\title{
LOS REGISTROS DE AUTORIDADES PERSONALES DE LA COMUNIDAD BIBLIOTECARIA REVISITADOS EN LA WEB SEMÁNTICA
}

\author{
Fernanda Garzón-Farinós* \\ Universidad Católica de Valencia San Vicente Mártir \\ Fernanda Peset Mancebo** \\ Universidad Politécnica de Valencia
}

\begin{abstract}
Resumen: Se plantea cómo la comunidad bibliotecaria gestiona información en paralelo con otros sectores profesionales del mundo web. Las herramientas y vocabularios controlados desarrollados en las bibliotecas aplican similares tecnologías que son el cimiento de la web semántica, las ontologías. Abordan los problemas que subyacen a la sobrecarga de información digital, ayudando a controlar y contextualizar los datos. Este texto propone un modelo de formatos de exportación que facilita el intercambio de datos de autoridades entre sistemas de información, en la biblioteca digital VESTIGIUM. Con este propósito se crea el patrón de la tabla autor, que cumple con las premisas de publicación de Linked Data (LD): los cuatro principios básicos definidos por BernersLee para la publicación de LD y así, asegura la visibilidad y contextualiza la información, creando un prototipo interoperable de datos biográficos.
\end{abstract}

Palabras clave: Control de autoridades; diccionarios biográficos; esquemas de metadatos; web semántica.

\begin{abstract}
Title: PERSONAL AUTHORITIES OF THE LIBRARIAN COMMUNITY OF PRACTICE REVISITED FOR SEMANTIC WEB.

Abstract: This document considers how the librarian community manages information in parallel with other professional sectors of the web environment. Similar technologies that are the basis of the semantic web (ontologies) were developed by librarians, who applying tools and controlled vocabularies. They address the underlying problems of digital information overload, helping to control and contextualize the data. This text proposes the modeling of export formats that facilitate the exchange of data of authorities between information systems, particularly in the digital library VESTIGIUM. With this objective, the 'authortable' pattern is created, which complies with the Linked Data (LD) publication premises: the four basic principles defined by Berners-Lee for the publication of LD. This ensures the contextualization and visibility of information by creating an interoperable prototype of biographical data.
\end{abstract}

Keywords: Name authority control; personal names; biographical dictionaries; metadata; semantic web.

Copyright: (C) 2018 Servicio de Publicaciones de la Universidad de Murcia (Spain). Este es un artículo de acceso abierto distribuido bajo los términos de la licencia Creative Commons Reconocimiento 4.0 Internacional (CC BY $4.0)$.

\section{INTRODUCCIÓN}

La comunidad bibliotecaria busca respuestas junto a otros sectores profesionales con el fin de gestionar el complejo mundo web, en especial cuando aparece la web semántica (Díaz-Valenzuela et al., 2013; Greenberg y Méndez, 2007; Leiva-Mederos et al., 2013). La web semántica requiere que la información sea entendida automáticamente por las máquinas, lo que supone un paso más allá en la interoperabilidad. El sueño de Tim BernersLee de una web de datos enlazados donde el intercambio, uso y reutilización de la información resulte fácil (BernersLee et al., 2001) está cada vez más próximo. La estandarización de metadatos enriquecidos con semántica y el uso de ontologías y lenguajes de marcado son la base de este nuevo entorno (Batjargal et al., 2013; Burke, 2009; Liangxian et al., 2012; W3C, 2015). En 2006, Berners-Lee propuso el modelo de datos Linked Open Data (LOD), planteando cuatro principios básicos para su publicación: 1) utilizar URI para identificar recursos de forma unívoca; b) usar URI HTTP para que los usuarios puedan acceder a la información del recurso; c) ofrecer información sobre los recursos utilizando RDF; d) incluir enlaces a otros URIs para facilitar el enlace entre diferentes datos distribuidos en la web (Berners-Lee, 2006; Gracy et al., 2013; Peset et al., 2011). Ciertos autores han percibido similitudes entre las bibliotecas y la web semántica. El deseo de interoperabilidad, de control universal de los recursos web y de reutilización en la web las acercan sin duda (Burke, 2009; Campbell, 2007; Greenberg, 2007; McCathieNevile y Méndez, 2007; Vila-Suero, 2016). Además, el uso de vocabularios controlados y ficheros normalizados de autoridades

\footnotetext{
*fernanda.garzon@ucv.es

***mpesetm@upv.es
}

Recibido: 01-08-2017; 2 $2^{\mathrm{a}}$ versión: 08-02-2018; aceptado: 09-02-2018.

GARZÓN FARINÓS, F. y PESET MANCEBO, F. Los registros de autoridades personales de la comunidad bibliotecaria revisitados en la web semántica. Anales de Documentación, 2018, vol. 21, $\mathrm{n}^{\mathrm{o}} 1$. Disponible en: http://dx.doi.org/10.6018/analesdoc.21.1.301521. 
-instrumentos habituales en nuestra profesión-son imprescindibles para enriquecer los datos en el escenario de la web semántica (Díaz-Valenzuela et al., 2013; Gracy et al., 2013; Harper y Tillet, 2007; Peset et al., 2011; Vila-Suero y Gómez-Pérez, 2013).

La iniciativa que pone en relación el mundo bibliotecario y la web semántica procede del W3Consortium, que en 2010 promueve el Library Linked Data Incubator Group. El grupo de trabajo analizó, entre otras actividades, la situación de los tipos y esquemas de metadatos, así como los estándares y protocolos de interoperabilidad que debían utilizarse para incluir los datos de bibliotecas como LD (World Wide Web Consortium (W3C), 2010). Su calado puede observarse por los casos que incluye, revisados por Daniel Vila Suero, de la Universidad Politécnica de Madrid: desde la British National Bibliography ${ }^{2}$ de la British Library ${ }^{3}$ a la versión de WorldCat $^{4}$ del Online Computer Library Center (OCLC) $)^{5}$. Se concluye que el uso de lenguajes documentales y vocabularios controlados publicados como LD facilita su interoperabilidad y reutilización en cualquier otro ámbito (Pastor-Sánchez, 2013).

Como hemos comentado, uno de los campos en que la comunidad web y la bibliotecaria coinciden es en el uso de ontologías (Figura 1).

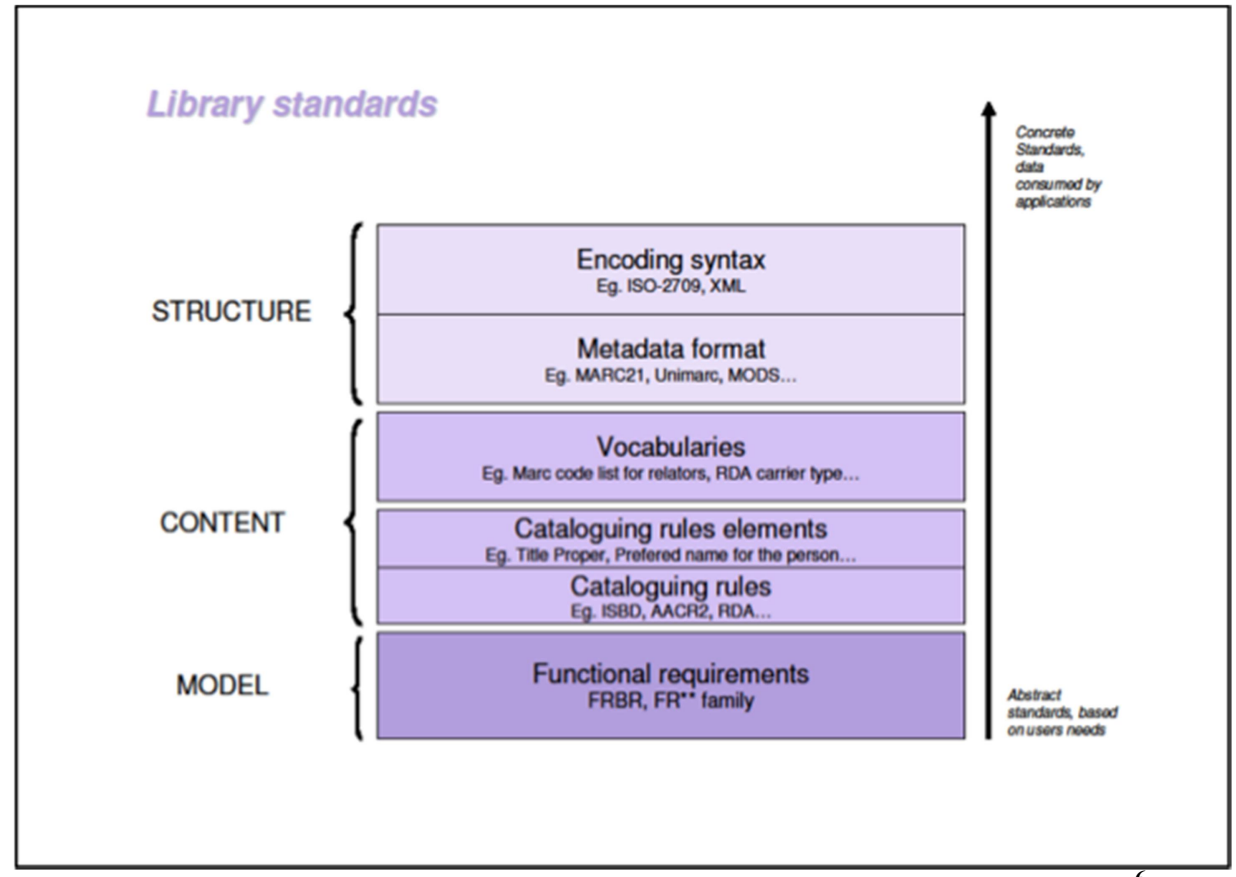

Figura 1. Niveles de las normativas utilizadas en bibliotecas. Fuente: LLD-XG'.

La larga experiencia de la comunidad Libraries, Archives and Museums (LAM) fue uno de los aspectos más apreciados desde el mencionado Incubator Group. En el Cluster Authority Data ${ }^{7}$ se presentaron estudios relacionados con ficheros de autoridad (W3C, 2011), tema del que nos ocupamos en este trabajo. Se incluyeron los casos de VIAF ${ }^{8}$, la Biblioteca de Alemania ${ }^{9}$, AuthorClaim ${ }^{10}$, AGRIS de FAO $^{11}$ e IraLIS $^{12}$, del grupo CIEPI, único caso español incorporado sobre datos de autoridades.

\section{OBJETIVOS Y METODOLOGÍA}

\subsection{Objetivos}

Nuestro trabajo tiene como objetivo describir la confluencia de ambas comunidades de práctica en el control de autoridades y su aplicación a la biblioteca digital VESTIGIUM. La biblioteca digital VESTIGIUM está orientada a recopilar la información de humanistas y científicos del siglo $\mathrm{XX}^{13}$, a modo de diccionario de autores que incluye tanto la biografía como sus trabajos. En este trabajo proponemos también un modelo de formatos de exportación que facilitan el intercambio de datos de autoridades entre sistemas de información con características similares, acordes con algunas iniciativas de la web semántica. De esta manera se podrán obtener datos y vocabularios semánticamente similares y a su vez consumir información externa. La visibilidad de esta información se consigue mediante el enriquecimiento semántico de los datos y su vinculación con fuentes externas. 


\subsection{Metodología}

La metodología para realizar este artículo está determinada por el análisis de las normativas en materia de autoridades personales en el ámbito de las bibliotecas y archivos para el control de autoridades: los Functional Requirements for Authority Data (FRAD), los Registros de Autoridad de Archivos relativos a Instituciones, Personas y Familias (ISAAR (CPF)), las Directrices para Registros de Autoridad y Referencias (GARR), MARC 21 Conciso para Datos de Autoridad, Metadata Authority Description (MADS) y MARCXML. Gracias a los resultados de un trabajo anterior (Garzón-Farinós, 2015; Garzón y Peset (en prensa)), que reflejan los atributos de la entidad persona de FRAD y sus conversiones a otros estándares de archivos, bibliotecas y web semántica, hemos podido crear para este texto los registros de autoridad y sus relaciones entre autoridades -de persona o corporativas- en los formatos: FRAD, ISAAR (CPF), GARR, MARC 21 Datos de Autoridad, MADS, MARCXML, EAC-CPF, Dublin Core RDF y FOAF ${ }^{14}$. En ese trabajo anterior ya está presentada la equivalencia con las procedentes del ámbito web: Encoded Archival Context - Corporate Bodies, Persons and Families (EAC-CPF), Dublin Core RDF y Friend to Friend (FOAF).

En este trabajo la conceptualización de FRAD y las equivalencias nos permiten construir los campos de la tabla autor. Para nuestro caso, VESTIGIUM, tomamos la codificación del formato MARC 21 Conciso para Datos de Autoridad, puesto que reúne la tradición precedente e incluye los conceptos de FRAD y las nuevas aplicaciones como RDA. En esta tabla se definen las entidades y los atributos necesarios para el control de autoridades, pero ofrecen a su vez información adicional de los autores de forma armónica con los desarrollos para la web.

Tras el desarrollo de la tabla de conversiones y equivalencias entre los formatos, se crearon los registros de autoridad de exportación y visualización en los formatos objeto de estudio.

Para ilustrarlo se utilizó la aplicación Editra ${ }^{15}$, un editor de texto que permite crear pseudocódigo, ofreciendo características y funciones para más de 60 lenguajes de programación, incluido el Extensible Markup Language (XML). Como ejemplo se muestra la biografía de Eduardo Primo Yúfera (Ayala, 2011). Se consultó el catálogo de la Biblioteca Nacional de España ${ }^{16}$, VIAF y LC Names Authorities File ${ }^{17}$ para encontrar las formas de referencia de envío "véase" (campo 400), "véase también" (campo 500) y encabezamientos en otras lenguas (campos 700) de nombre personal.

\section{ANTECEDENTES NORMATIVOS}

A continuación, se describen las normativas utilizadas para la realización de este trabajo. GARR es una de las primeras pautas para la descripción de autoridades y su forma en el mundo de las bibliotecas (IFLA, 2004). La familia de los MARC apareció con posterioridad en la década de los 60/70 para automatizar los catálogos de bibliotecas. Ha continuado hasta nuestros días con la última adaptación de MARC 21 que incluye los postulados de los Functional Requirements for Bibliographic Records (FRBR) basados en el modelo entidad relación (Orduña-Malea, 2008; VilaSuero, 2016), apreciándose como un instrumento esencial para el intercambio de información en entornos automatizados (Library of Congress, 2017; Lam, 2002; Veve, 2009; Vila-Suero, 2016). En este contexto, RDA también proporciona directrices e instrucciones sobre la descripción de recursos digitales, cubriendo todos los tipos de contenido y medios de comunicación. Se basa en los modelos conceptuales desarrollados por FRBR y FRAD. Una de sus principales características es la flexibilidad para ser utilizados en la web (Joint Steering Committee, 2010; Picco y Ortiz Repiso, 2012).

A este mismo movimiento de acercamiento al modelo entidad relación pertenecen las normativas ISAAR, FRAD y MADS. ISAAR describe las entidades creadoras de archivos y la información de contexto de los creadores, con la posibilidad de compartir y vincular datos e información con los catálogos y sistemas descriptivos más allá del ámbito archivístico (Buiza, 2004; Consejo Internacional de Archivos, 2004). FRAD desarrolla un modelo conceptual para los registros de autoridad cuyo principal objetivo consiste en compartir datos con sectores como archivos, museos y otras organizaciones que gestionan derechos de autor (Patton, 2009; Vila-Suero, 2016). Por último, MADS desarrolla un esquema de codificación de datos en formato MARC 21 Conciso para Datos de Autoridad a RDF para su uso en aplicaciones de la web semántica y proyectos de LD (Library of Congress, 2017; Vila-Suero, 2016).

Uno de los aspectos más característicos de los últimos estándares mencionados para el ámbito de la comunidad LAM y de los utilizados para web es su capacidad de interrelacionar ítems, tanto en el propio sistema como con información externa. En este contexto, EAC-CPF facilita la codificación y la comunicación de registros de autoridad en XML, según las normas establecidas por ISAAR (CPF) (Society of American Archivistics, 2010). Dublin Core RDF permite establecer un sistema normalizado para la descripción de documentos digitales que aparecen en la web mediante un conjunto de etiquetas, indiferentemente de su formato (Dublin Core Metadata Iniciative, 2017). 
Asimismo, FOAF es un lenguaje desarrollado en el contexto del W3C para compartir y enlazar información que describe personas, sus actividades y las relaciones con otras personas (Brian y Dodds, 2005; Brickley y Miller, 2010).

\section{RESULTADOS}

En este apartado indicaremos la propuesta desarrollada para el sistema de información VESTIGIUM. En primer lugar, se presenta la Tabla I que contempla las relaciones genéricas entre autoridades y sus relaciones con otros nombres de personas, familias y entidades. A continuación se propone el modelo de tabla autor en formato MARC 21 Conciso para Datos de Autoridad. Posteriormente se describirá el formato ficha de presentación de las biografías y los registros confeccionados en los estándares utilizados para el intercambio de información de la tabla autor.

\subsection{Equivalencias genéricas}

La Tabla I representa las relaciones entre las autoridades y sus equivalencias entre diferentes normativas para posteriormente desarrollar una aplicación que refleje las correspondencias de personas con otros nombres de personas, familias y entidades. Este sistema relaciona las variantes que se producen a lo largo del tiempo, como nombres anteriores o posteriores, especialmente en los corporativos. Las relaciones más comunes son las que enlazan las variantes de nombres por utilizar otros idiomas, otras escrituras alternativas u otras reglas.

En la primera columna se detallan las relaciones a nivel general entre los tipos de entidad. Siguiendo la estructura de FRAD se dibujan los paralelismos para la relación entre persona y persona, la relación entre persona y familia y relación entre persona y entidad corporativa, incluyendo el nombre específico por el que la entidad es conocida.

La relación entre persona y persona (FRAD) abarca las forma(s) autorizada(s) del nombre (campo 100), formas paralelas del nombre, formas normalizadas del nombre según otras reglas y otras formas del nombre lingüísticas (campos 100, 400, 500, 700, $700 \$ a$ q, $700 \$ d$ y $700 \$ b \$ c$ ) de MARC 21. Lo que supone, por tanto, que es un campo con las variantes. El siguiente tipo trata las relaciones en la estructura de referencias del registro de autoridad, es decir, las referencias propiamente dichas como el "véase" o "úsese por". Por una parte, incluye relaciones específicas de persona y, por otra, un nombre específico por el que la entidad es conocida. Esta entidad comprende la relación nombre de persona y nombre de persona -relación de nombre anterior, relación de nombre posterior, relación de forma lingüística alternativa, relación de otras formas variantes de nombre-. El último tipo de relaciones se refiere a la entidad punto de acceso controlado. Ese mismo modo se refleja en las otras dos categorías: relación entre persona y familia (campo 500) y relación entre persona y entidad corporativa (campo 510). Ambos son utilizados para indicar la pertenencia, ya sea a la familia o a la entidad, aunque para esta última, la variabilidad de los nombres corporativos a lo largo del tiempo le confiere mayor interés. 
LOS REGISTROS DE AUTORIDADES PERSONALES DE LA COMUNIDAD BIBLIOTECARIA

\begin{tabular}{|c|c|c|c|c|c|c|c|c|}
\hline FRAD & ISAAR(CPF) & EAC-CPF & GARR & MARC 21 & MADS & $\begin{array}{l}\text { DUBLIN } \\
\text { CORE RDF }\end{array}$ & MARCXML & FOAF \\
\hline $\begin{array}{l}\text { relación persona } \\
\rightarrow \text { Persona } \\
\text { relación nombre } \\
\text { de persona } \rightarrow \\
\text { Nombre } \\
\text { persona } \\
\text { relación punto de } \\
\text { acceso } \\
\text { controlado } \rightarrow \\
\text { Punto de } \\
\text { acceso } \\
\text { controlado }\end{array}$ & $\begin{array}{l}\begin{array}{l}5.1 .2 \text { Forma(s) } \\
\text { autorizada(s) del } \\
\text { nombre }\end{array} \\
\begin{array}{l}5.1 .3 \quad \text { Formas } \\
\text { paralelas } \\
\text { nombre del }\end{array} \\
\begin{array}{l}\text { 5.1.4 Formas } \\
\text { normalizadas del } \\
\text { nombre según } \\
\text { otras reglas }\end{array} \\
\begin{array}{l}\text { 5.1.5 Otras formas } \\
\text { del nombre }\end{array}\end{array}$ & $\begin{array}{l}<\text { nameEntry }>0 \\
<\text { nameEntryParallel }>\text { con } \\
<\text { authorizedForm }>\text { / } \\
\text { <nameEntry }> \\
\text { <nameEntryParallel }>\text { con } \\
<\text { alternativeForm }>\text { / } \\
\text { <nameEntry }> \\
<\text { nameEntryParallel }>\text {. } \\
\text { Irá acompañado de } \\
<\text { authorizedForm }>\end{array}$ & $\begin{array}{l}1.1 \\
(1.1 .1 .2 .) \\
\text { Área de } \\
\text { asiento } \\
\text { autorizado } \\
\text { de persona } \\
\text { / } \\
1.3 \text {. Área } \\
\text { de trazado } \\
\text { de } \\
\text { referencia } \\
\text { de } \\
\text { "véase"/ } \\
\text { 1.4. Área } \\
\text { de trazado } \\
\text { de } \\
\text { referencia } \\
\text { de "véase } \\
\text { también" }\end{array}$ & $\begin{array}{l}100 / 400 / 500 / \\
700,700 \quad \$ a \$ q \\
700 \$ d / 700 \$ b \$ c\end{array}$ & 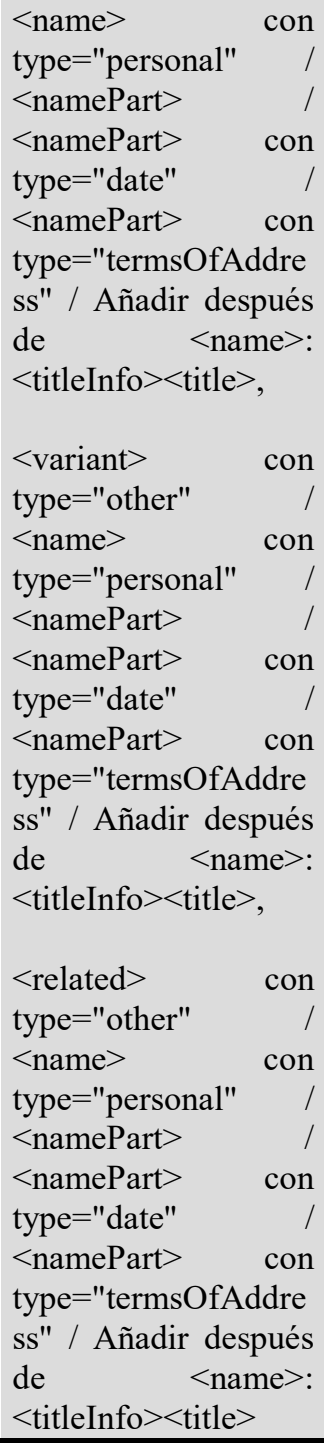 & $\begin{array}{l}\text { dc:title } \\
\text { dc:relation }\end{array}$ & $\begin{array}{l}\text { <datafield } \\
\text { tag="100" / "400" } \\
\text { / "500" / "700" } \\
\text { ind1="0","1" } \\
\text { ind2=" "> / } \\
\text { <datafield } \\
\text { tag="400" } \\
\text { ind1="0","1" } \\
\text { ind2=" "> / } \\
\text { <datafield } \\
\text { tag="500" } \\
\text { ind1="0","1" } \\
\text { ind2=" "> con } \\
<\text { subfield } \\
\text { code="a">,<subfi } \\
\text { eld code="b">, } \\
\text { <subfield } \\
\text { code="c">,<subfi } \\
\text { eld code="d">, } \\
\text { <subfield } \\
\text { code="e">, } \\
\text { <subfield } \\
\text { code="h">, } \\
<\text { subfield } \\
\text { code="q"> }\end{array}$ & $<$ foaf:name $>$ \\
\hline
\end{tabular}




\begin{tabular}{|c|c|c|c|c|c|c|c|c|}
\hline 6 & & & & & \multicolumn{4}{|c|}{ FERNANDA GARZÓN Y FERNANDA PESET } \\
\hline FRAD & ISAAR(CPF) & EAC-CPF & GARR & MARC 21 & MADS & $\begin{array}{l}\text { DUBLIN } \\
\text { CORE RDF }\end{array}$ & MARCXML & FOAF \\
\hline $\begin{array}{lr}\text { Relaciones } & \text { entre } \\
\text { persona } & \rightarrow \\
\text { Familia } & \end{array}$ & $\begin{array}{l}\text { 5.3.2 Naturaleza } \\
\text { de la relación }\end{array}$ & $\begin{array}{l}<\text { cpfRelation } \\
\text { cpfRelationType }="[\text { value }] \\
">\end{array}$ & $\begin{array}{l}\text { 1.4. Área } \\
\text { de trazado } \\
\text { de } \\
\text { referencia } \\
\text { de "véase } \\
\text { también" }\end{array}$ & 500 & $\begin{array}{lr}<\text { related }> & \text { con } \\
\text { type="other" } & / \\
<\text { name }> & \text { con } \\
\text { type="personal" } & / \\
<\text { namePart> } & / \\
<\text { namePart> } & \text { con } \\
\text { type="date" } & / \\
<\text { namePart> con } \\
\text { type="termsOfAddre } \\
\text { ss" / Añadir después } \\
\text { de } \quad<\text { name>: } \\
<\text { titleInfo }><\text { title> }\end{array}$ & dc:relation & $\begin{array}{l}\text { <datafield } \\
\text { tag="500" } \\
\text { ind1="0","1" } \\
\text { ind2=" "> con } \\
\text { <subfield } \\
\text { code="a">,<subfi } \\
\text { eld code="b">, } \\
\text { <subfield } \\
\text { code="c">,<subfi } \\
\text { eld code="d">, } \\
\text { <subfield } \\
\text { code="e">, } \\
<\text { subfield } \\
\text { code="h">, } \\
\text { <subfield } \\
\text { code="q"> }\end{array}$ & $<$ foaf:familyName $>$ \\
\hline $\begin{array}{l}\text { Relaciones entre } \\
\text { personas } \rightarrow \\
\text { Entidad } \\
\text { corporativa }\end{array}$ & $\begin{array}{l}\text { 5.3.2 Naturaleza } \\
\text { de la relación }\end{array}$ & $\begin{array}{l}<\text { cpfRelation } \\
\text { cpfRelationType }="[\text { value }] \\
">\end{array}$ & $\begin{array}{l}\text { 1.4. Área } \\
\text { de trazado } \\
\text { de } \\
\text { referencia } \\
\text { de "véase } \\
\text { también" }\end{array}$ & 510 & 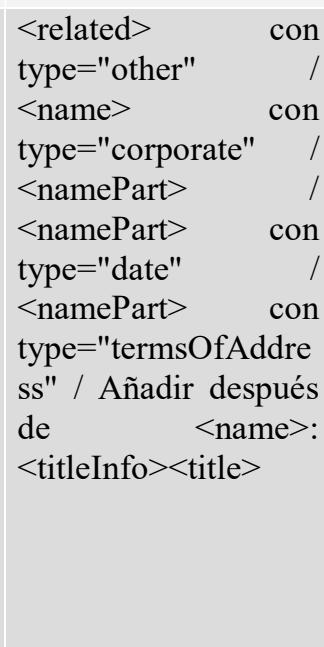 & dc:relation & $\begin{array}{l}\text { <datafield } \\
\text { tag="510" } \\
\text { ind1="0","1" } \\
\text { ind2=" "> con } \\
\text { <subfield } \\
\text { code="a">,<subfi } \\
\text { eld code="b">, } \\
\text { <subfield } \\
\text { code="c">,<subfi } \\
\text { eld code="d">, } \\
\text { <subfield } \\
\text { code="e">, } \\
\text { <subfield } \\
\text { code="h">, } \\
\text { <subfield } \\
\text { code="q"> }\end{array}$ & $<$ foaf:knows $>$ \\
\hline
\end{tabular}

Tabla I. Relaciones entre autoridades (de persona o corporativas). Fuente: Elaboración propia. 


\subsection{VESTIGIUM y los campos de la tabla autor}

Tomando como base las equivalencias de la Tabla I se han desarrollado los módulos biográficos, de exportación y de visualización de los registros de autoridad, que se verán en la siguiente sección. Estos últimos presentan los siguientes formatos: MARC 21 Conciso para Datos de Autoridad, MARCXML, Dublin Core RDF, Metadata Authority Description (MADS), Directrices para Registros de Autoridad y Referencias (GARR), ISAAR (CPF) y FOAF.

Entre ellos destacan los que se aplican a la web semántica, que subyace en nuestra biblioteca digital VESTIGIUM, como veremos más adelante.

En la Tabla II se contemplan los campos seleccionados MARC 21 para realizar las biografías de las personalidades integradas en VESTIGIUM. Especifica los identificadores de etiqueta para nuestra base de datos, la descripción del campo y el correspondiente campo MARC 21. También indica si el campo es repetible y si es visible en el formato ficha de los registros de autoridad. En caso necesario, se han explicitado los códigos de subcampo que van a ser más utilizados, por ejemplo, las fechas de nacimiento y fallecimiento.

Cada registro de autoridad MARC 21 se compone del encabezamiento principal (campo 100), las variantes de nombre (campo 400), las relaciones con otros nombres y las relaciones con otras personalidades e instituciones (campo 5XX), así como la equivalencia en otros idiomas (campo 700). A ellos hemos añadido los nuevos campos específicos para los atributos de persona procedentes de RDA, como detalla la Tabla II. 


\begin{tabular}{|c|c|c|c|c|c|c|c|}
\hline IDENTIFICADOR & MARC 21 & IDENTIFICADOR & MARC 21 & IDENTIFICADOR & MARC 21 & IDENTIFICADOR & MARC 21 \\
\hline Cabecera & $\begin{array}{l}\text { No repetible } \\
\text { No visible }\end{array}$ & $\begin{array}{l}\text { Fecha de nacimiento } \\
\text { Fecha fallecimiento }\end{array}$ & $\begin{array}{l}\text { 046* } \\
\text { Repetible. } \\
\text { Visible \$f: } \\
\text { nac./\$g: fallec. }\end{array}$ & $\begin{array}{l}\text { Dirección/s (residencia, } \\
\text { trabajo, e-mail) }\end{array}$ & $\begin{array}{l}371^{*} \\
\text { Repetible } \\
\text { Visible }\end{array}$ & $\begin{array}{l}\text { Información sobre } \\
\text { formas autorizadas de } \\
\text { nombres }\end{array}$ & $\begin{array}{l}664 \\
\text { Repetible } \\
\text { No Visible }\end{array}$ \\
\hline Campos de control & & $\begin{array}{l}\text { Nombre personal - } \\
\text { forma autorizada del } \\
\text { nombre- }\end{array}$ & $\begin{array}{l}\mathbf{1 0 0} \\
\text { No repetible } \\
\text { No visible }\end{array}$ & Campo de actividad & $\begin{array}{l}\mathbf{3 7 2} * \\
\text { Repetible } \\
\text { Visible }\end{array}$ & $\begin{array}{l}\text { Información sobre } \\
\text { formas autorizadas de } \\
\text { encabezamientos } \\
\text { nombres }\end{array}$ & $\begin{array}{l}\mathbf{6 6 3} \\
\text { Repetible } \\
\text { Visible }\end{array}$ \\
\hline $\begin{array}{l}\text { Número de control } \\
\text { de registro }\end{array}$ & $\begin{array}{l}\mathbf{0 0 1} \\
\text { No repetible } \\
\text { No visible }\end{array}$ & Tipo de contenido & $\begin{array}{l}336^{*} \\
\text { Repetible } \\
\text { No visible }\end{array}$ & Afiliación & $\begin{array}{l}\mathbf{3 7 3} * \\
\text { Repetible } \\
\text { Visible }\end{array}$ & $\begin{array}{l}\text { Nota general } \quad-\text { sin } \\
\text { despliegue al público- }\end{array}$ & $\begin{array}{l}667 \\
\text { Repetible } \\
\text { No visible }\end{array}$ \\
\hline $\begin{array}{l}\text { Identificador del } \\
\text { número de control }\end{array}$ & $\begin{array}{l}\mathbf{0 0 3} \\
\text { No repetible } \\
\text { No visible }\end{array}$ & $\begin{array}{l}\text { Otros atributos de la } \\
\text { persona o entidad }\end{array}$ & $\begin{array}{l}\mathbf{3 6 8 \$ d *} \\
\text { Repetible } \\
\text { Visible }\end{array}$ & Ocupación (profesión) & $\begin{array}{l}\mathbf{3 7 4} * \\
\text { Repetible } \\
\text { Visible }\end{array}$ & $\begin{array}{l}\text { Fuente en la que se } \\
\text { localizaron los datos }\end{array}$ & $\begin{array}{l}\mathbf{6 7 0} \\
\text { Repetible } \\
\text { Visible }\end{array}$ \\
\hline $\begin{array}{l}\text { Fecha y hora de la } \\
\text { última transacción }\end{array}$ & $\begin{array}{l}\mathbf{0 0 5} \\
\text { No repetible } \\
\text { No visible }\end{array}$ & $\begin{array}{l}\text { Lugares } \\
\text { vinculación }\end{array}$ & $\begin{array}{l}370 * \\
\text { Repetible } \\
\text { Visible }\end{array}$ & Género & $\begin{array}{l}375^{*} \\
\text { Repetible } \\
\text { No visible }\end{array}$ & $\begin{array}{l}\text { Fuente en la que no se } \\
\text { localizaron los datos }\end{array}$ & $\begin{array}{l}675 \\
\text { Repetible } \\
\text { No visible }\end{array}$ \\
\hline Información general & $\begin{array}{l}\mathbf{0 0 8} \\
\text { No repetible } \\
\text { No visible }\end{array}$ & Nacimiento & $\begin{array}{l}\mathbf{3 7 0} \$ \mathbf{a} * \\
\text { No repetible } \\
\text { Visible }\end{array}$ & $\begin{array}{l}\text { Información de la } \\
\text { familia }\end{array}$ & $\begin{array}{l}376^{*} \\
\text { Repetible } \\
\text { Visible }\end{array}$ & $\begin{array}{l}\text { Datos biográficos y/o } \\
\text { históricos }\end{array}$ & $\begin{array}{l}678 \\
\text { No repetible } \\
\text { Visible }\end{array}$ \\
\hline $\begin{array}{l}\text { Otro identificador } \\
\text { Normalizado }\end{array}$ & $\begin{array}{l}\mathbf{0 2 4} \\
\text { No repetible } \\
\text { No visible }\end{array}$ & Fallecimiento & $\begin{array}{l}370 \$ b^{*} \\
\text { No repetible } \\
\text { Visible }\end{array}$ & Lengua asociada & $\begin{array}{l}\mathbf{3 7 7} * \\
\text { Repetible } \\
\text { No visible }\end{array}$ & $\begin{array}{l}\text { Nota general } \quad \text { con } \\
\text { despliegue al público- }\end{array}$ & $\begin{array}{l}\mathbf{6 8 0} \\
\text { Repetible } \\
\text { Visible }\end{array}$ \\
\hline Campos de números & y códigos & País & $\begin{array}{l}370 \$ c^{*} \\
\text { Repetible } \\
\text { No Visible }\end{array}$ & $\begin{array}{l}\text { Puntos de acceso a las } \\
\text { variantes del nombre - } \\
\text { relación véase- }\end{array}$ & $\begin{array}{l}\mathbf{4 0 0} \\
\text { Repetible } \\
\text { Visible }\end{array}$ & $\begin{array}{l}\text { Puntos de acceso a } \\
\text { encabezamientos en } \\
\text { otras lenguas }\end{array}$ & $\begin{array}{l}\mathbf{7 0 0} \\
\text { Repetible } \\
\text { Visible }\end{array}$ \\
\hline $\begin{array}{l}\text { Número de control } \\
\text { del sistema }\end{array}$ & $\begin{array}{l}\mathbf{0 3 5} \\
\text { No repetible } \\
\text { No visible }\end{array}$ & Residencia & $\begin{array}{l}\text { 370\$e* } \\
\text { Repetible } \\
\text { Visible }\end{array}$ & $\begin{array}{l}\text { Campos de acceso a las } \\
\text { variantes del nombre - } \\
\text { relación véase además- }\end{array}$ & $\begin{array}{l}\mathbf{5 0 0} \\
\text { Repetible } \\
\text { Visible }\end{array}$ & $\begin{array}{l}\text { Localización y accesos } \\
\text { electrónicos }\end{array}$ & $\begin{array}{l}\mathbf{8 5 6 *} \\
\text { Repetible } \\
\text { Visible }\end{array}$ \\
\hline Centro catalogador & $\begin{array}{l}\mathbf{0 4 0} \\
\text { No repetible } \\
\text { No visible }\end{array}$ & Otro lugar asociado & $\begin{array}{l}\text { 370\$f* } \\
\text { Repetible } \\
\text { Visible }\end{array}$ & $\begin{array}{l}\text { Campos de acceso a las } \\
\text { variantes de } \\
\text { instituciones -relación } \\
\text { véase además- }\end{array}$ & $\begin{array}{l}\mathbf{5 1 0} \\
\text { Repetible } \\
\text { Visible }\end{array}$ & Publicaciones & $\begin{array}{l}\mathbf{6 7 0} \\
\text { Repetible } \\
\text { Visible }\end{array}$ \\
\hline
\end{tabular}




\subsection{Visualización de los registros}

La visualización de la información, como se sabe, es independiente de las normativas que se utilicen. En cualquier caso, recordemos que nuestro sistema VESTIGIUM ha utilizado de base el formato MARC 21, incluyendo además algunos metadatos de Dublin Core y MADS. Mediante este procedimiento, y gracias a utilizar un Content Manager System (CMS) que soporta módulos de descripción y de exportación en RDF, la biblioteca VESTIGIUM se integra en el ámbito de los datos enlazados.

La Figura 2 muestra el formato ficha del autor Eduardo Primo Yúfera tal como se visualiza en VESTIGIUM. Como puede verse, el formato MARC 21 es suficientemente completo para cualquier registro de autoridad. Por ejemplo, permite recoger las principales ocupaciones que ha desarrollado a lo largo de su vida. La ocupación aparece con enlaces, de manera que permite navegar por las biografías de los autores que se hayan codificado con las mismas profesiones (Fig. 2, superior izquierda), a modo de etiquetas.

En la Figura 2 (superior derecha) aparece la actividad laboral (campo 373 \$e) que, siguiendo FRAD, incluye los organismos con los que se ha relacionado este autor, con el valor añadido de poder navegar hacia otras autoridades relacionadas mediante enlaces. Uno de los puntos fuertes de utilizar esta normativa es que potencia la interoperabilidad y la captura de información externa. MARC 21 mantiene el clásico trabajo con autoridades sobre las variaciones de las formas de los nombres que han de ser registradas. En este caso, la referencia de "véase" y referencia de "véase además" corresponden a los campos 400, 500 y 700 (Figura 2, inferior derecha); incluso las relaciones que establece con otras personas o entidades se contemplan en el campo 5XX.

Otro aspecto destacable es el soporte multilingüe del MARC 21. El nombre en otras lenguas de la referencia "véase además" (campo 700) permite registrar los encabezamientos en otros idiomas (Figura 2, inferior derecha). El código de subcampo $\$ 0$ incluye el número de registro de la base de datos externa de la que se tomen los datos y el subcampo \$1 la lengua del registro. En el ejemplo el nombre de autor personal Eduardo Primo Yúfera ha sido tomado de un registro OCLC, lo que muestra la incorporación de fuentes externas con MARC 21:

$$
\text { \$wa\$aPrimo Yúfera, E. \$c(Eduardo)\$IInglés\$O(OCoLC)oca03519974 }
$$

A través de los trazados de referencia del campo $5 \mathrm{XX}^{18}$ es posible describir las relaciones e influencias entre personas (500) e instituciones (510). En nuestra Figura 2 (inferior izquierda) son reflejadas en los apartados relaciones personales y relaciones institucionales. Las URIs que se expresan en la parte inferior izquierda se registran en el subcampo \$4 y corresponden a otras ontologías como Schema.org, FOAF, Relationship (Davis y Vitiello, 2010) o Dbpedia. Es decir, facilitan la descripción de información biográfica y la definición de las relaciones entre personas. Con el subcampo \$i de MARC 21 se indica la relación entre el encabezamiento principal y el encabezamiento relacionado. En los siguientes ejemplos encontramos las relaciones de persona a persona y de persona a institución.

\section{Tipo de relación FRAD}

Persona a persona

Persona a institución

\section{Código MARC 21}

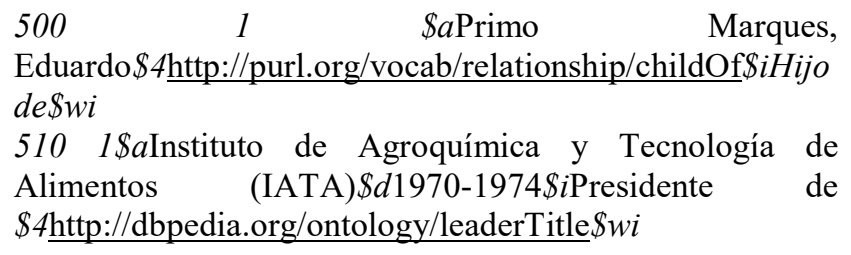

Un último recurso de este modelo de biblioteca digital semántica para integrar los registros biográficos en el mundo web es el campo 856. En él se registra información externa sobre el autor, a través de páginas web y otros sistemas de información que utilizan LD: LC Names Authorities File, FAST Linked Data ${ }^{19}$, VIAF, WorldCat Identities y Dbpedia ${ }^{20}$. Las fuentes utilizadas variarán según la información localizada en cada una de ellas. 


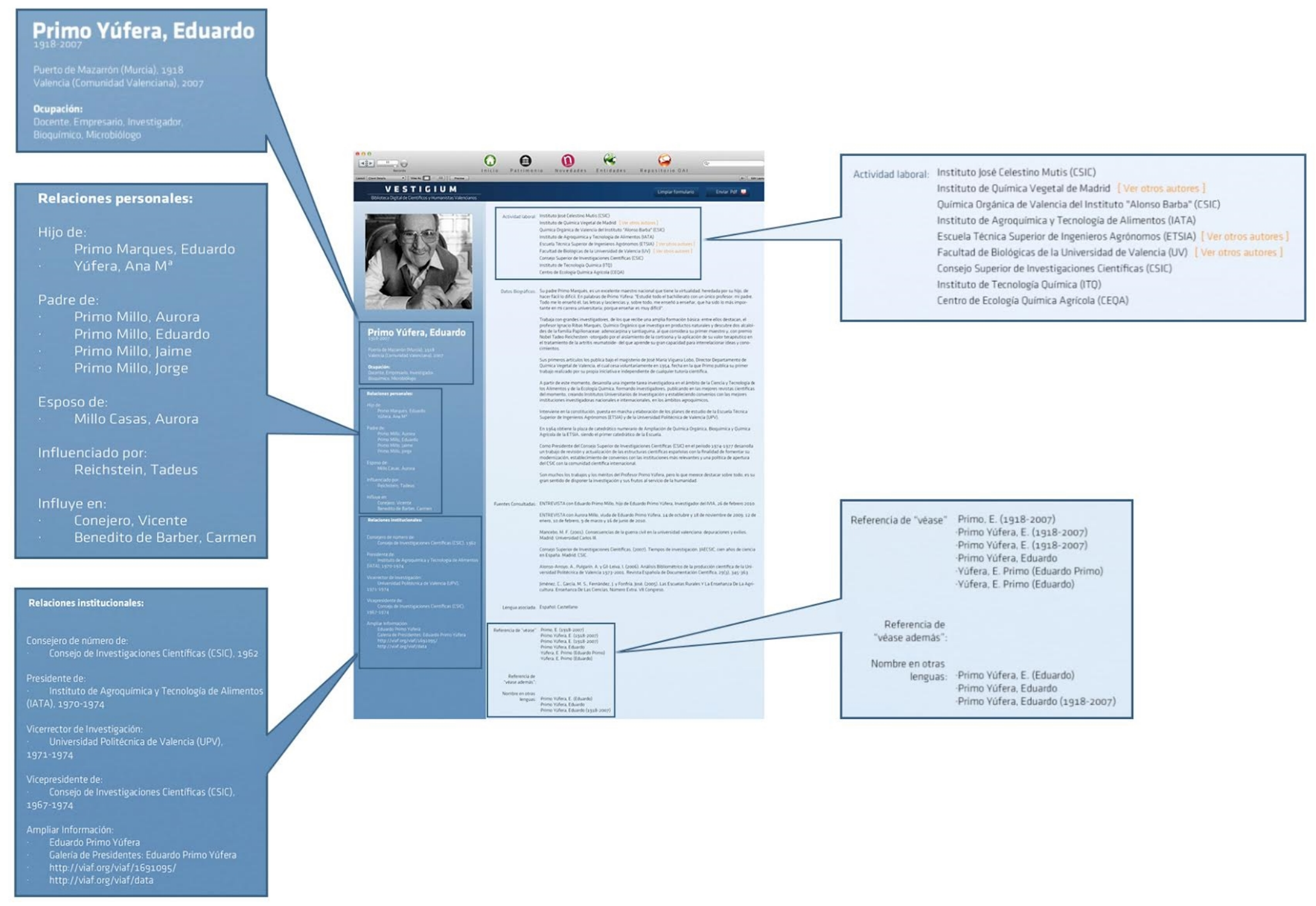

Figura 2. Formato beta ficha de autor en VESTIGIUM. Fuente: Elaboración propia. 
Por último, destacamos la utilidad que se ha incorporado en nuestro sistema VESTIGIUM gracias a la aportación teórica que refleja este artículo, es decir las equivalencias expresadas anteriormente. VESTIGIUM cuenta con la posibilidad de exportar la información a otros formatos. A partir de la Tabla I se han creado los formatos que facilitan el intercambio de información entre diferentes sistemas.

Los siguientes registros de autoridad en MARCXML y formato FOAF (Figuras 3 y 4 respectivamente), tomados como ejemplo entre las nueve normativas analizadas, lo muestran. Como se puede observar, el campo 024 refleja los URIs de diversas fuentes, donde aparecen enlaces a los encabezamientos de autoridad en otras fuentes de información como VIAF y LC Names Authorities File.

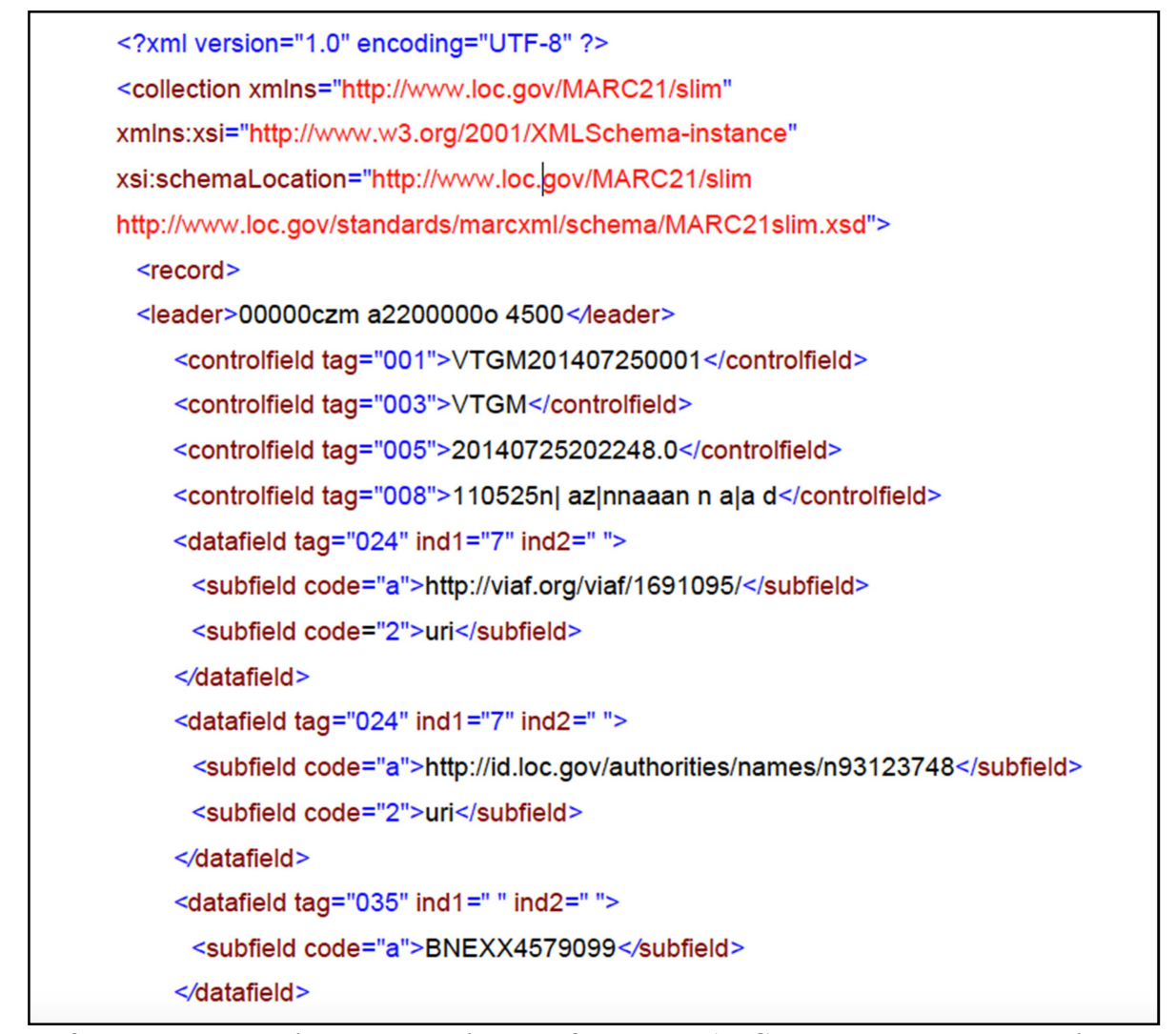

Figura 3. Extracto de registro de autoridad en formato MARCXML. Fuente: Elaboración propia.

La Figura 4 muestra el registro de autoridad en FOAF con las relaciones entre las formas no autorizadas y otras variantes no elegidas. También ofrece la relación con otras personas e instituciones, a través de la propiedad foaf:name y foaf:Organization, de la clase foaf:Person. 


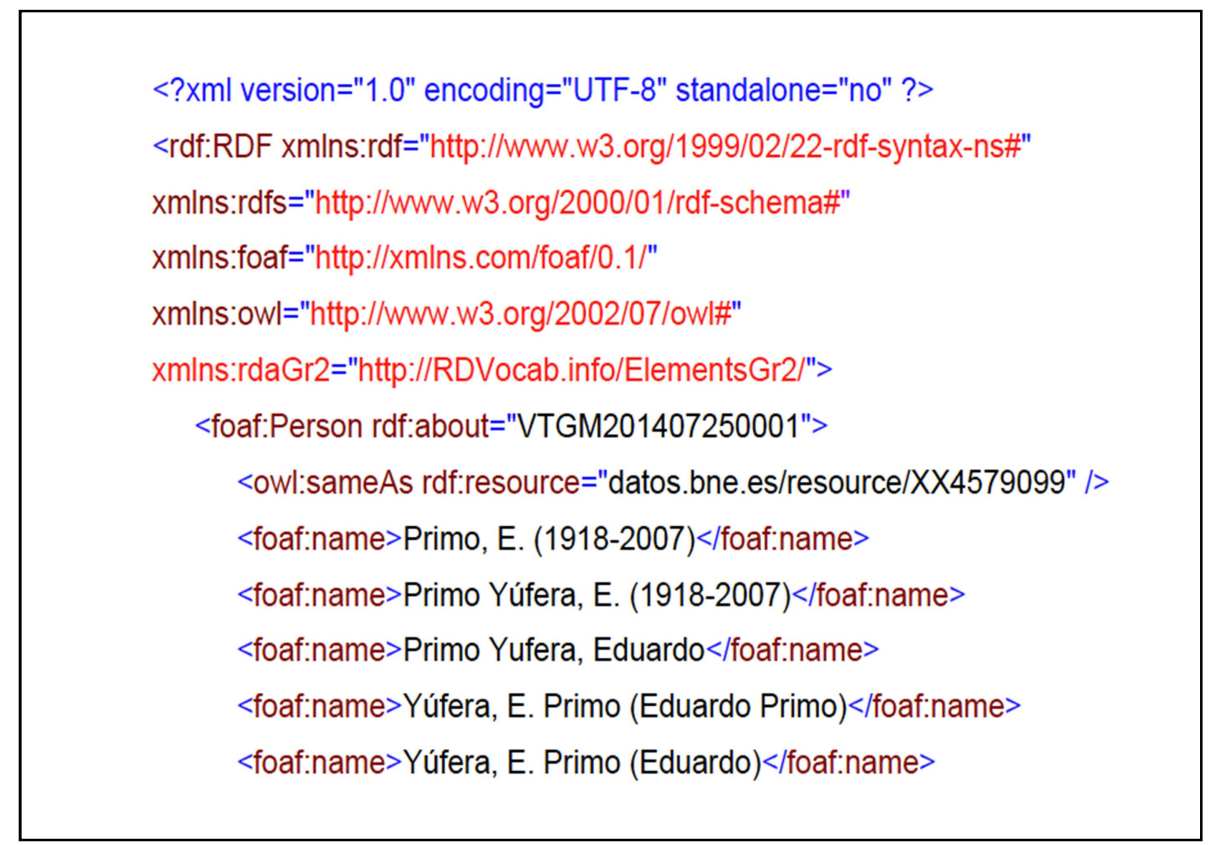

Figura 4. Extracto de registro de autoridad en formato FOAF. Fuente: Elaboración propia.

\section{CONCLUSIONES}

La web semántica es un intento de mejorar la recuperación de la información e interoperabilidad entre máquinas, utilizando el marco de descripción RDF, que hemos visto que se incorpora en algún CMS como Drupal y que es utilizado por VESTIGIUM. El concepto de enriquecimiento de la información resulta de extrema actualidad: reutiliza los datos de fuentes externas, y además permite intercambiar información, de modo que las personas y las máquinas puedan interpretar la pertenencia a una clase o instancia en diferentes entornos semánticos.

El diseño de la tabla autor sirve de prototipo para impulsar la interoperabilidad de datos biográficos, asegurando la visibilidad a escala internacional. El modelo de datos propuesto en dicha tabla cumple con los cuatro principios básicos definidos por Berners-Lee y el informe de OCLC (2014) para la publicación de LD.

Este estudio proporciona la estructura de la información biográfica de personajes a través del diseño de la tabla autor y sus formatos de exportación en VESTIGIUM. Tal y como se evidencia en este trabajo, es perfectamente posible hacer confluir la comunidad LAM con el desarrollo de la web con relativa facilidad. En definitiva, esta investigación ha recogido por una parte las tablas de equivalencias entre las diferentes normativas, ha creado una tabla autor en la normativa más completa -MARC 21 Conciso para Datos de Autoridad- y, por último, hemos mostrado cómo se han implementado en el sistema VESTIGIUM con los datos descriptivos, con las relaciones y con los formatos de exportación que hacen posible la interoperabilidad entre máquinas.

Los beneficios de utilizar normativa armonizada con la web semántica son numerosos. Por ejemplo, mejora el proceso de obtención de enlaces entre entidades semánticamente similares o relacionadas. También facilita el uso de vocabularios de valores y conjuntos de elementos de metadatos o conjuntos de datos que provienen de diferentes fuentes. Por último, mejora la difusión de este tipo de datos de autoridades ya que el uso de protocolos de producción y consumo de datos mediante LD supone un avance. Nuestro modelo, en definitiva, fija unas normas comunes a muchas fuentes, por lo que facilita el trabajo de los desarrolladores utilizando una sola herramienta para múltiples fuentes que exponen sus datos en LD.

\section{NOTAS}

\footnotetext{
${ }^{1}<$ http://www.w3.org/2005/Incubator/lld/>.

$2<$ http://bnb.bl.uk>.

$3<$ http://www.bl.uk/>.

$4<$ http://www.worldcat.org/identities/>.

${ }^{5}<$ https://www.oclc.org/>.
} 


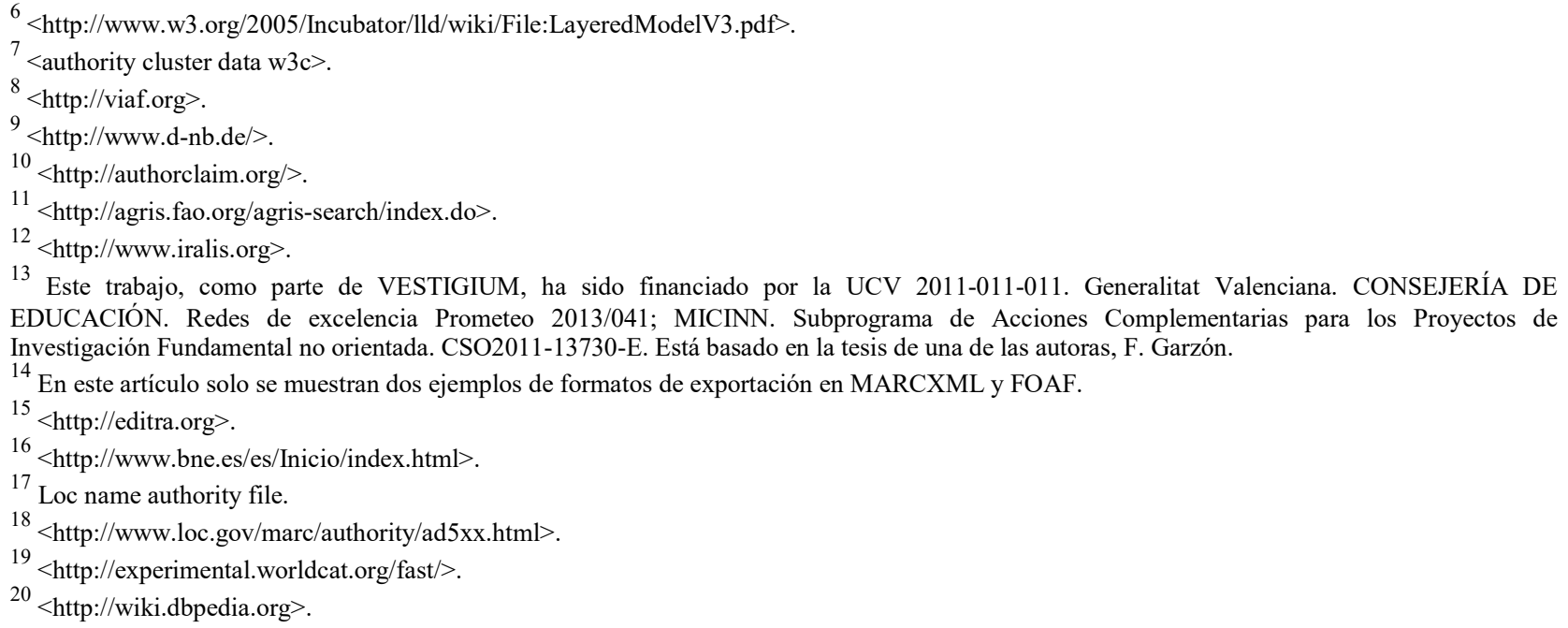

\section{NORMATIVA UTILIZADA}

BIBFRAME. https://www.loc.gov/bibframe/ DUBLIN CORE. http://dublincore.org EAC-CPF. http://eac.staatsbibliothek-berlin.de/ FOAF. http://xmlns.com/foaf/spec/ FRAD. http://www.ifla.org/publications/ifla-series-on-bibliographic-control-34

GARR. http://www.ifla.org/files/cataloguing/garr/garr-es.pdf

ISAAR (CPF). http://www.archivonacional.go.cr/pdf/ISAAR2-ES.pdf

MADS. http://www.loc.gov/standards/mads/

MARC 21 Format for Authority Data.

http://www.loc.gov/marc/authority/ecadhome.html

MARC 21 Conciso para Datos de Autoridad.

https://www.loc.gov/marc/authority/ecadspa.html

MARC 21 XML. http://www.loc.gov/standards/marcxml/

RDF. http://www.w3.org/RDF/

RELATIONSHIP. http://vocab.org/relationship/

\section{BIBLIOGRAFÍA}

AYALA GASCÓN, M.; ALEIXANDRE BENAVENT, R. y GANDÍA BALAGUER, A. Eduardo Primo Yúfera, Founder of Revista de Agroquímica y Tecnología de Alimentos and pioneer on Food Science and Technology research in Spain. Food Science and Technology International, 2011, vol. 17, nº 6, p. 549-556.

BATJARGAL, B. et al. Linked data driven multilingual access to diverse Japanese Ukiyo-e databases by generating links dynamically. Literary and Linguistic Computing, 2013, vol. 28, n 4, p. 522-530.

BERNERS-LEE, T.; HENDLER, J. y LASSILA, O. The Semantic Web. A new form of Web content that is meaningful to computers will unleash a revolution of new possibilities. Scientific American, 2001, vol. 284, $\mathrm{n}^{\mathrm{o}}$ 5, p. 34-44.

BERNERS-LEE. Linked Data. Actualizado 13 de junio de 2009. Disponible en: $<$ https://www.w3.org/DesignIssues/LinkedData.html $>$. [Consulta: 1 de abril de 2017].

BRIAN, K. y DODDS, L. Using FOAF to Support Community Building [en línea]. The European Journal for the Informatics Professional, 2005, vol. $\quad$ VI, $\mathrm{n}^{\mathrm{o}} \quad 5 . \quad$ Disponible en: $<$ http://opus.bath.ac.uk/423/1/BKelly_Upgrade_10_2005.pdf $>$. [Consulta: 14 de abril de 2017].

BRICKLEY, D. y MILLER, L. FOAF at a glance [en línea]. In: FOAF Vocabulary Specification 0.98. Namespace Document 9 August 2010. Marco Polo Edition. Disponible en: $<$ http://xmlns.com/foaf/spec/>. [Consulta: 1 de julio de 2017].

BUIZZA, P. Bibliographic Control and Authority Control form Paris Principles to the Present. Cataloging \& Classification Quartely, 2004, $\mathrm{n}^{\mathrm{o}}$ 38, p. 117-133.

BURKE, M. The semantic web and the digital library. Aslib Proceedings, 2009, vol. 61, n 3, p. 316-322.

CAMPBELL, G. The birth of the New Web: a Foucauldian Reading of the Semantic Web. Cataloging \& Classification Quarterly, 2007, vol. 43, n 3/4, p. 9-20. 
DAVIS, I. y VITIELLO, E. RELATIONSHIP: A vocabulary for describing relationships between people. 2010 (editorial change) [en línea]. Disponible en: <http://vocab.org/relationship/>. [Consulta: 25 de mayo de 2017].

DÍAZ-VALENZUELA, I.; MARTIN-BAUTISTA, M.; VILA, M.A. y CAMPAÑA, J.R. An automatic system for identifying authorities in digital libraries. Expert Systems with Applications, 2013, vol. 40, n 10, p. 3994-4002.

DUBLIN CORE METADATA INITIATIVE. Dublin Core Metadata Terms. Actualizado 2017 [en línea]. Disponible en: <http://dublincore.org/documents/2012/06/14/dcmi-terms/>. [Consulta: 25 de octubre de 2015].

GARZÓN FARINÓS, M.F. El registro de autoridades personales tras la aparición del web. Valencia: Universidad Politécnica de Valencia, 2015.

GARZÓN FARINÓS, M.F. y PESET, F. Normativas interoperables sobre control de autoridades para bibliotecas digitales semánticas (artículo aceptado, en prensa). Investigación Bibliotecológica: Archivonomía, Bibliotecología e Información, 2018, $\mathrm{n}^{\mathrm{0}} 77$.

GRACY, K.F.; ZENG, M.L. y SKIRVIN, L. Exploring methods to improve access to Music resources by aligning library Data with Linked Data: A report of methodologies and preliminary findings. Journal of the American Society for Information Science and Technology, 2013, vol. 64, nº 10, p. 2078-2099.

GREENBERG, J. Advancing the Semantic Web via Library Functions. Cataloging \& Classification Quarterly. 2007, vol. $43, n^{\circ} 3 / 4$, p. 203-227.

GREENBERG, J. y MÉNDEZ, E. Introduction: toward a more library-like Web via Semantic Knitting. Cataloging \& Classification Quarterly, 2007, vol. 43, no 3/4, p. 1-8.

HARPER, C.A. y TILLET, B.B. Library of Congress controlled vocabularies and their application to the Semantic Web. Cataloging \& Classification Quarterly, 2007, vol. 43, n 3/4, p. 47-68.

IFLA. Directrices para registros de autoridad y referencias: anteriormente denominadas "Directrices para los asientos de autoridad y referencia" recomendadas por el Grupo de Trabajo para un Sistema Internacional de Autoridades, aprobadas por los Comités Permanentes de la Sección de Catalogación de la IFLA y de la Sección de Tecnología de la Información de la IFLA. $2^{\mathrm{a}}$ ed. revisada por el Grupo de Trabajo de la IFLA para la Revisión de las GARE. Madrid: Ministerio de Cultura, Secretaría General Técnica, 2004 [en línea]. Disponible en: $<$ http://www.ifla.org/files/cataloguing/garr/garr-es.pdf $>$. [Consulta: 29 de enero de 2017].

JOINT STEERING COMMITE. RDA: Resource Description and Access [en línea]. Chicago: American Library Association. Actualizado 13 de abril de 2015. Disponible en: <http://www.rda-jsc.org/rda.html $>$. [Consulta: 29 de enero de 2017].

LAM, Ki-Tat. XML and global name Access control. OCLC Systems and Services, 2002, vol. 18, n 2, p. 88-96.

LEIVA-MEDEROS, A.; SENSO, J.A.; DOMÍNGUEZ-VELASCO, S. y HÍPOLA, P. Authoris: a tool for authority control in the semantic web. Library Hi Tech, 2013, vol. 31, n 3, p. 536-553.

LIANGXIAN, D.; JUNXIA, Q. y PENGFEI, G. The Application of Semantics Web in Digital Library Knowledge Management. Physics Procedia, 2012, vol. 24, Part C, p. 2180-2186.

LIBRARY OF CONGRESS. MADS: Metadata Authority Description. MADS 2.0 [en línea]. Washington DC: Library of Congress. Actualizado 17 de abril de 2017. Disponible en: <http://www.loc.gov/standards/mads/>. [Consulta: 20 de julio de 2017].

LIBRARY OF CONGRESS. Network Development and Marc Standards Office. 2016. Marc 21 Format for Authority Data. 1999 Edition Update No. 1 (October 2000) through Update No. 24 (May 2017) [en línea]. Actualizada 24 de mayo de 2017. Disponible en: <http://www.loc.gov/marc/authority/ecadhome.html $>$. [Consulta: 20 de julio de 2017].

LIBRARY OF CONGRESS. Network Development and MARC Standards Office. Marc 21 Format for Authority Data. 1999 Edition Update No. 1 (October 2000) through Update No. 21 (September 2015) [en línea]. Washington DC: Biblioteca del Congreso. Actualizado septiembre de 2015. Disponible en: $<$ http://www.loc.gov/marc/authority/ecadhome.html $>$. [Consulta: 22 de febrero de 2017].

McCATHIENEVILE, C. y MÉNDEZ, E. Library Cards for the $21^{\text {st }}$ Century. Cataloging \& Classification Quarterly, 2007, vol. 43, no 3/4, p. 21-45.

OCLC. Results from OCLC Research International Linked data survey for implementation [en línea]. Actualizado 2015. Disponible en: <http://www.oclc.org/research/news/2014/09-19.html>. [Consulta: 15 de enero de 2017].

ORDUÑA-MALEA, E. Análisis de los FRBR en la ejecución de tareas genéricas en catálogos compartidos. Anales de documentación, 2008, nº 11, p. 129-144.

PASTOR-SÁNCHEZ, J.-A. Ontologías de control de autoridades en el ámbito de los datos abiertos enlazados. Anuario ThinkEPI, 2013, p. 184-188.

PATTON, Glenn E. (ed.). Requisitos Funcionales de los Datos de Autoridad (FRAD): un modelo conceptual. Informe Final [en línea]. Madrid: Biblioteca Nacional, 2009. Disponible en: $<$ http://www.ifla.org/files/cataloguing/frad/frad_2009-es.pdf $>$ [Consulta: 16 de febrero de 2017].

PESET, F.; FERRER-SAPENA, A. y SUBIRATS-COLL, I. Open data y Linked open data: su impacto en el área de bibliotecas y documentación. El profesional de la información, 2011, vol. 20 marzo-abril, no 2, p. 165-173. 
PICCO, P. y ORTIZ REPISO, V. RDA, el nuevo código de catalogación: cambios y desafíos para su aplicación. Revista Española de Documentación Científica, 2012, vol. 35, no 1 enero-marzo, p. 145-173.

SOCIETY OF AMERICAN ARCHIVISTICS. EAC - Encoded Archival Context, Corporate, Persons and Families [en línea]. Disponible en: <http://eac.staatsbibliothek-berlin.de/>. [Consulta: 19 de marzo de 2017].

VILA-SUERO, D. A framework for ontology-based library data generation, access and exploitation. [tesis doctoral inédita]. Madrid: Universidad Politécnica de Madrid, 2016.

VILA-SUERO, D. y GÓMEZ-PÉREZ, A. datos.bne.es and MARiMbA: an insight into Library Linked Data. Library Hi Tech, 2013, vol. 31, nº 4, p. 575-601.

W3C. Cluster Authority Data. Uses cases / Case studies. W3C Incubator Group Report 25 October 2011 [en línea]. Disponible en: <http://www.w3.org/2005/Incubator/lld/XGR-lld-usecase-20111025/>. [Consulta: 18 de octubre de 2016].

W3C. Incubator activity - W3C Library Linked Data Incubator Group. Actualizado 29 de junio de 2010 [en línea]. Disponible en: <http://www.w3.org/2005/Incubator/lld/>. [Consulta: 18 de octubre de 2016].

W3C. Semantic Web. Actualizado 2015 [en línea]. Disponible en: <http://www.w3.org/standards/semanticweb/> [Consulta: 18 de octubre de 2016]. 